\title{
$\underline{P-46}$
}

\section{The Antioxidant Activity of Myrmecodia Platytyrea}

\author{
N. F. Mohamad Haris, Mizaton H. H* I. Abdul Wahab, T. Ponto and A. Adam
}

Faculty of Pharmacy, UiTM, 42300 Puncak Alam, Selangor Darul Ehsan; E-mail: haydenbeck7@gmail.com

The Myrmecodia platytyrea extracts (hexane, dichloromethane, ethyl acetate and methanol) were screened using 2, 2-diphenyl1-picrylhydrazyl (DPPH) modified method against ascorbic acid (AA) and Trolox as the positive controls. The ethyl acetate (EtOAc) extract was found potent compared to the other extracts with $\mathrm{EC}_{50}$ value of $32.91 \pm 2.23 \mu \mathrm{g} / \mathrm{mL}$, whereas the $\mathrm{EC}_{50}$ value of AA and trolox were $4.56 \pm 0.41 \mu \mathrm{g} / \mathrm{mL}$ and $5.35 \pm 0.66 \mu \mathrm{g} / \mathrm{mL}$, respectively. Then, an automated gradient system of a preparative medium performance liquid chromatography (MPLC) technique was utilized to fractionate the potent EtOAc extract (6 gram). Later, the collected fractions were combined according to the TLC profiles and reduced to 8 fractions (F1 $\mathrm{F} 8$ ). DPPH scavenging assay was carried out on the fractions and the $\mathrm{EC}_{50}$ value of $\mathrm{F} 5$ was found lower with $\mathrm{EC}_{50}$ value of $21.57 \pm 1.40 \mu \mathrm{g} / \mathrm{mL}$. This indicated that F5 was potent in scavenging free radical and this may be due to the rich phenolic constituents which are known as good antioxidants. Next, the ferrous ion chelating (FIC) ability was tested on the crude EtOAc extract and the result was inactive compared to ethylenediaminetetraacetic acid (EDTA, $\mathrm{IC}_{50}=23.15 \pm 2.26 \mu \mathrm{g} / \mathrm{mL}$ ), the standard used in this assay. The EtOAc fractions (F1 - F8) also underwent FIC test and surprisingly, the fractions (F1 and F2) were active with $\mathrm{IC}_{50}$ values of $293.30 \pm 19.86 \mu \mathrm{g} / \mathrm{mL}$ and $152.74 \pm 9.95 \mu \mathrm{g} / \mathrm{mL}$, respectively. Based on the data, M. platytyrea possessed promising antioxidant activity. Thus, this plant indeed is a medicinal plant, not only claimed by the community web portals but could be proven with the scientific evidences. Successive work would include the identification of the major chemical entities in this extract. This effort would append to more information of the former isolation of stigmasterol and a phenolic from the hexane extract of this plant.

Keywords: DPPH, FIC, Myrmecodia platytyrea. 